$\cos ^{2} \theta \sin ^{2} \theta$. However, for $\mathrm{Fe}^{3+}$ ions in $\mathrm{MgO}$ the cubic field causes a small admixing of states ${ }^{1}$ and consequently, a slight deviation of the transition probability from a simple $\cos ^{2} \theta \sin ^{2} \theta$ dependence. Therefore, exact wave functions have been used in computing the theoretical angular variation.

A quadrupole spin operator cannot induce transitions between pure $-1 / 2$ and $+1 / 2$ states, and the near-zero ultrasonic effect observed on this transition for all three ions is further evidence for the quadrupole interaction.

The authors would like to thank Dr. G. D. Wat- kins for helpful discussions, and Mr. T. G. Kazyaka for his aid in taking the data.

${ }^{1}$ W. Low, Paramagnetic Resonance in Solids (Academic Press, New York, 1960).

${ }^{2}$ R. D. Mattuck and M. W. P. Strandberg, Phys. Rev. Letters $\underline{3}, 369$ (1959).

${ }^{3}$ R. D. Mattuck and M. W. P. Strandberg, Phys. Rev. 119, 1204 (1960).

${ }^{4}$ E. H. Jacobsen, N. S. Shiren, and E。 B. Tucker, Phys. Rev. Letters 3,81 (1959).

${ }^{5}$ E. B. Tucker, Bull. Am. Phys. Soc. $\underline{5}, 157$ (1960).

${ }^{6}$ N. S. Shiren, Bull. Am. Phys. Soc. $\underline{5}, 343$ (1960).

\title{
POPULATION INVERSION AND CONTINUOUS OPTICAL MASER OSCILLATION IN A GAS DISCHARGE CONTAINING A He-Ne MIXTURE
}

\author{
A. Javan, W. R. Bennett, Jr., and D. R. Herriott
} Bell Telephone Laboratories, Murray Hill, New Jersey (Received December 30, 1960)

This Letter is intended to give a summary of the results of experimental determinations of several physical properties of a gaseous discharge consisting of a $\mathrm{He}-\mathrm{Ne}$ mixture which have led to the successful operation of a continuouswave maser at five different wavelengths in the near-infrared. Population inversions are achieved between several $\mathrm{Ne}$ levels by means of excitation transfer from the metastable $\mathrm{He}\left(2^{3} S\right)$ to the $2 s$ levels of $\mathrm{Ne}^{1,2}$ The maser oscillation takes place in a narrow beam with a diameter of 0.45 inch. The power in our strongest beam at the wavelength $11530 \mathrm{~A}$ is 15 milliwatts. The measured linewidth is in the range of 10 to $80 \mathrm{kc} / \mathrm{sec}$. The angular spread of the beam is less than one minute of arc.

In the present system, the $\mathrm{He}\left(2^{3} S\right)$ metastables are used as carriers of energy to excite the levels of Ne. Due to the resonant nature of this process, the excitation cross section is large only to those levels of Ne which fall in energy within a few $k T$ of that of the $\mathrm{He}\left(2^{3} \mathrm{~S}\right)$. Figure 1 gives the pertinent energy levels of $\mathrm{He}$ and $\mathrm{Ne}$. As may be seen from Fig. 1, ${ }^{3}$ the energy separations between the $\mathrm{He}\left(2^{3} S\right)$ and $\mathrm{Ne}(2 s)$ states are small enough to insure an appreciable degree of transfer to the $2 s$ states; however, the energy gap between the $2{ }^{3} S$ and $2 p$ levels is much too large to permit direct population of the $2 p$ states in the collision process. Although the $2 s_{2}$ and $2 s_{4}$ levels

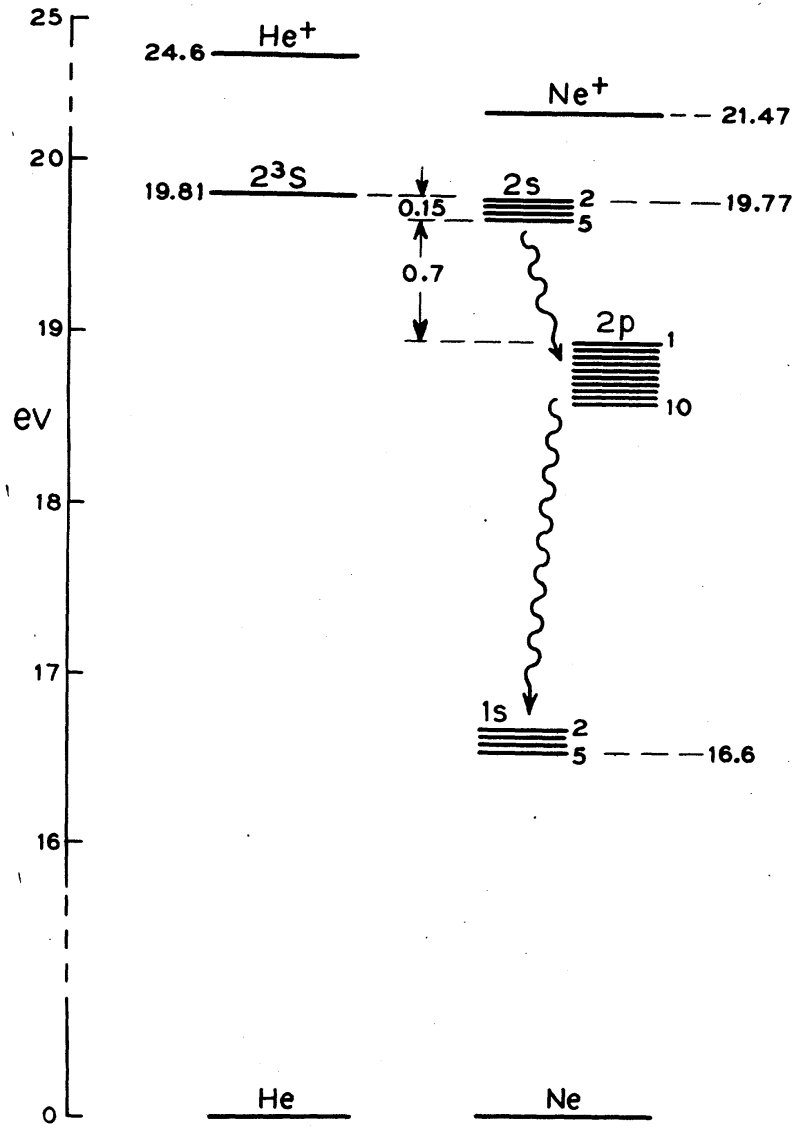

FIG. 1. Energy level diagram of He and Ne atoms. 
of $\mathrm{Ne}$ may radiate to the $\mathrm{Ne}$ ground state, in the limit of complete resonance trapping their lifetimes are determined primarily by radiative decay to the $2 p$ levels. Under this condition, the lifetimes of all of the $2 s$ levels are about one order of magnitude longer than those of the corresponding $2 p$ states. (The decay of the $2 p$ levels is due to their radiative decay to the $1 s$ levels.) Thus population inversions may be obtained on each of the thirty allowed $2 s \rightarrow 2 p$ transitions (see below).

In order to determine the transfer cross section and spurious sources of excitation of $\mathrm{Ne}$ levels, the afterglow of a pulsed $\mathrm{rf}$ discharge containing varying amounts of $\mathrm{He}$ and $\mathrm{Ne}$ was studied in detail. The time variation of the densities of the $2 s$ and $2 p$ levels was observed by the fluorescent decay of these levels using appropriate isolated transitions. The time variation of the $2{ }^{3} S$ density was determined through the absorption of the $2{ }^{3} \mathrm{P} \rightarrow 2{ }^{3} \mathrm{~S}$ He transition.

The conditions were found under which the dominant mode of excitation of $\mathrm{Ne}$ levels arose from the $\mathrm{He}\left(2^{3} S\right)$ transfer. Among other sources of excitation of the Ne levels it was found that inelastic collisions of energetic electrons with Ne metastables ${ }^{2}$ (i.e., the $1 s$ levels $^{4}$ ) were by far the most important. It should be noted that certain amounts of $2 p$ excitation, arising from sources other than $2 s-2 p$ cascade, can be tolerated without upsetting the presence of a population inversion. For instance, let us consider the population inversion between the $2 s_{5}$ and $2 p_{9}$ levels. The $2 p_{9}$ is optically connected to only one of the $2 s$ levels, namely the $2 s_{5}$. The Einstein $A$ coefficient for the $2 s_{5} \rightarrow 2 p_{9}$ transition was meas ured and found to be at least 25 times smaller than the rate of decay of the $2 p_{9}$ level to within an accuracy of $20 \%$. (This measurement was done by means of two independent techniques. The first was a spectroscopic technique to be described in a later publication. The second is discussed below.) This means that even an order of magnitude added excitation of $2 p_{9}$ over that due to the cascade from the $2 s_{5}$ still yields a population of the $2 s_{5}$ levels larger than twice the population of the $2 p_{9}$. In view of this effect, the adjustment of the electron density was found to be most critical.

In the afterglow, the energetic electrons thermalize within 10 to $20 \mu \mathrm{sec}$. The excitation transfer from $\mathrm{He}\left(2^{3} S\right)$, however, continues until the time when all of the $2^{3} S$ states are quenched. This effect gives rise to a decay of the $2 s$ levels identical to the time variation of the $\mathrm{He}\left(2^{3} S\right)$. The afterglow of the $2 p$ levels also shows an identical decay due to the cascade transition from the $2 s$ levels. Our observations indicate that the time variations of the $2{ }^{3} S, 2 s, 2 p$ levels in the afterglow are indeed identical to a high degree of accuracy. The possibility of the direct trans fer from the $2{ }^{3} S$ to the $2 p$ levels was ruled out by a process of relative intensity measurement at a fixed time in the afterglow to be described elsewhere.

Study of the fluorescent decay rate of the $2 s$ and $2 p$ levels as a function of several Ne pressures gave a total velocity-averaged inelastic cross section of $\langle\sigma\rangle=(3.7 \pm 0.5) \times 10^{-17} \mathrm{~cm}^{2}$ for destroying $\mathrm{He}\left(2^{3} S\right)$ metastables in two-body collisions with $\mathrm{Ne}$ ground-state atoms at $300^{\circ} \mathrm{K}$. This cross section can safely be assigned to the excitation transfer to the $2 s$ levels of Ne since we have been able to show that other sources of destruction of the $\mathrm{He}\left(2^{3} \mathrm{~S}\right)$ by $\mathrm{Ne}$ ground-state atoms such as the one leading ${ }^{5}$ to a formation of $\mathrm{HeNe}^{+}$are negligible. Our cross-section measurement at the same time furnishes the value of the diffusion coefficient of $2{ }^{3} \mathrm{~S}$ He metastables. Our result is in excellent agreement with the value of $\left(470 \pm 25 \mathrm{~cm}^{2} / \mathrm{sec}\right)$ determined by Phelps.

The radiative lifetimes of the $2 s$ and $2 p$ levels were determined using techniques described previously. ${ }^{7}$ Our tentative values of the lifetimes of the $2 s_{2}, 2 s_{3}, 2 s_{4}$, and $2 s_{5}$ fall in the range from about 100 to $200 \mathrm{~m} \mu \mathrm{sec}$. Our results for the $2 p$ levels are in general agreement with Ladenberg's ${ }^{8}$ results. These measurements are still in progress and will be reported more fully at a later date. Each of the $2 s$ levels decays into several of the ten $2 p$ levels. The measured and estimated branching ratios for the transitions originating from each of the $2 s$ levels, together with their total rate of radiative decay obtained from the above lifetimes, give the values of the individual Einstein $A$ coefficients connecting the various $2 s$ and $2 p$ levels.

From the early stages of this experiment we were able to observe single-pass amplification of a transmitted signal at an infrared frequency where the inverted population was expected. Several electronic detection schemes were devised for this purpose. One of these schemes responded only during a small portion of the time and was in synchronism with the rf pulsed discharge. This technique enabled us to observe a larger value of amplification in the afterglow than during the glow; the latter is to be expected 
since the added excitation of the $2 p$ levels by electron impact is absent in the afterglow. However, in addition to extreme difficulties encountered in the interpretation of the results, the gain measurement alone was not sufficient to shed insight into the nature of the physical processes involved in the discharge.

In view of the above considerations, we were able to determine an optimum condition for the maser action prior to the observation of oscillation as described below.

The continuous-wave maser oscillation is observed in a discharge containing $0.1 \mathrm{~mm} \mathrm{Hg}$ of $\mathrm{Ne}$ and $1 \mathrm{~mm} \mathrm{Hg}$ of He. The optical feedback ${ }^{9}$ is obtained through high-reflectance Fabry-Perot plates placed within the gas chamber. This chamber consists of a long quartz tube with an inside diameter of $1.5 \mathrm{~cm}$ and length of $80 \mathrm{~cm}$. The quartz tube is terminated at each end with a larger metal chamber containing the high-reflectance plates. Flexible bellows are used in the end chambers to allow external mechanical tuning of the Fabry-Perot plates. Two optically flat windows at the extremes of this system are provided to allow the maser beam to leave the instrument undistorted. The separation of the plates is one meter. The discharge is excited by means of external electrodes using a 28 $\mathrm{Mc} / \mathrm{sec}$ generator. The estimated power dissipation is around 50 watts.

The high reflectance is achieved by means of 13-layer evaporated dielectric films on the hundredth wavelength flat surface of the fused silica plates. The reflectance is $98.9 \%$ with $0.3 \%$ transmission in the wavelength range $11000 \mathrm{~A}$ to $12000 \mathrm{~A}$. Outside of this range of wavelength the reflectance decreases rapidly.

The initial adjustment of the plates prior to starting of the discharge is done using an autocollimator. We have observed oscillations at the following wavelengths: $11180,11530,11600$, 11990 , and $12070 \mathrm{~A}$. The strongest oscillation occurs at $11530 \mathrm{~A}$ with an output power of $15 \mathrm{mw}$.

An image converter tube is used visually and with a camera to observe and record structure of the beam. Two types of beam patterns have been studied.

A cross section of the emergent beam can be intercepted by the photocathode of the image converter and the intensity distribution recorded. This near-field pattern is shown in the two upper photographs of Fig. 2 for two Fabry-Perot plate angles.

The intensity pattern that the beam would form

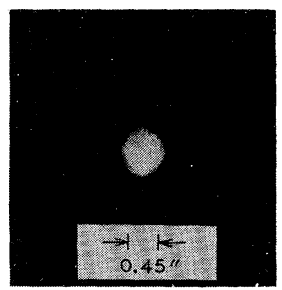

PLATES PARALLEL

PATTERN IN MASER CAVITY
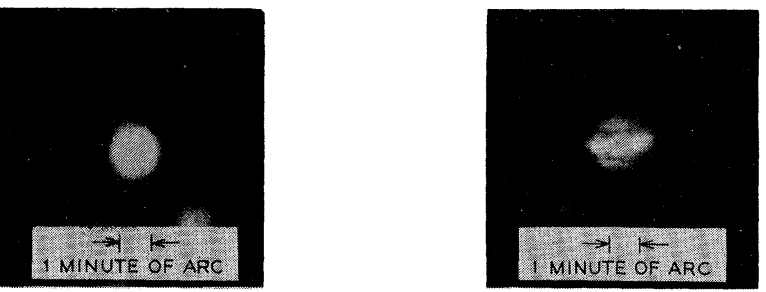

PLATES PARALLEL
ANGULAR DISTRIBUTION IN BEAM

FIG. 2. Far-field and near-field patterns from maser beam at two plate angles.

at an infinite distance can be observed by imaging the maser beam to a point and observing the distribution of light in this image with an image converter microscope. The intensity distribution in this image represents the angular distribution in the maser beam. The two lower pictures in Fig. 2 show this far-field pattern at the indicated plate positions. The structures seen in the nearand far-field patterns would indicate the existence of several modes at a finite plate angle. The patterns shown in Fig. 2 were made at constant exposure and are therefore greatly overexposed by the increased intensity with parallel plates. The half-intensity width of the parallel plate output is less than one minute of arc.

The phase variation over the area of the beam was studied by placing a double or multiple slit in the beam and recording the diffraction pattern. A nine-slit mask of 0.050 -in. spacing and $0.002-$ in. slit width covering the full 0.45 -in. aperture of the beam results in a diffraction pattern shown in Fig. 3. The minima are very low, indicating small phase variation over the aperture. A double-slit pattern at 0.0175 -in. is also shown.

Since the linewidth of the maser oscillation is many orders of magnitude narrower than the resolution of the best available spectrometers or 

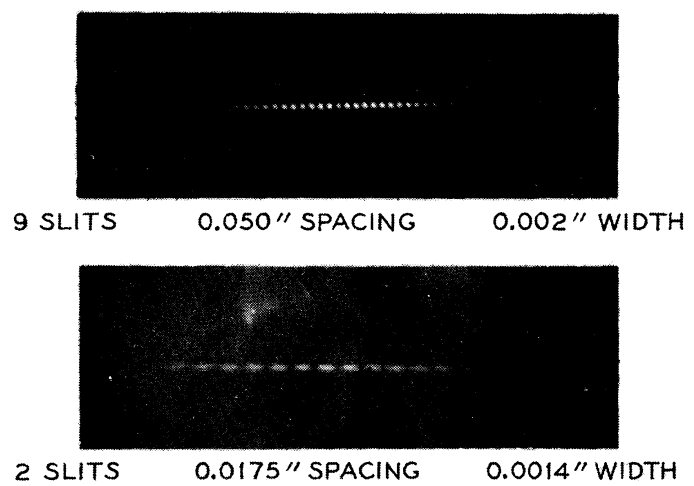

FIG. 3. Far-field diffraction patterns obtained by inserting multiple slits in the maser beam.

interferometers, standard optical techniques could not be used to measure the linewidth. Of the several possible methods examined, the most practical was found to be one based on analysis of the Fourier spectrum observed through a photomultiplier tube of the maser output. The photomultiplier is essentially a perfect squarelaw detector and should respond to beat frequencies. Hence, one can observe the spectral distribution obtained by beating all of the components of the line. The exact width of the original line determined from this distribution is independent of detailed assumptions on the line shape to within a factor of about two, providing the original line is not multiply peaked. In practice, this spectral distribution will be superimposed on top of the flat spectrum obtained from shot noise in the photomultiplier tube, and the latter may be used to check the bandwidth of the system.

The system was first checked by observing the spectrum from an intense line emitted in an ordinary discharge tube using a spectrum analyzer. The latter was flat within the frequency range of our analyzer, covering from $100 \mathrm{cps}$ to $13 \mathrm{Mc} / \mathrm{sec}$. Using a grating spectrometer to isolate the strong line at $11530 \mathrm{~A}$, the maser output was next examined. Our initial observations indicated a strong signal starting at zero frequency and extending to a half-width of about $100 \mathrm{kc} / \mathrm{sec}$. Further examination showed the presence of an additional series of anharmonically related beats centered about frequencies other than zero. The location of these beats depended critically on the Fabry-Perot plate alignment. As the angular alignment of the plates was changed from 0 to 3 seconds, the beats became more widely spaced and weaker in intensity. Several possible spurious sources of beats were carefully examined and found not to be responsible for the observed signal. The possibility of "blocking oscillation" of the maser giving rise to an amplitude modulation of the output signal can be ruled out by the follow ing observation: The peak frequency of a beat note is only a function of the plate adjustment and is independent of the factor by which the maser gain exceeds the oscillation threshold. The most plausible explanation found was that the beats arose from simultaneous oscillation in several different modes separated by frequencies in the $\mathrm{Mc} / \mathrm{sec}$ range. Such a conclusion is not in disagreement with detailed calculations of the oscillatory modes by Fox and Li. ${ }^{10} \mathrm{~A}$ distinct

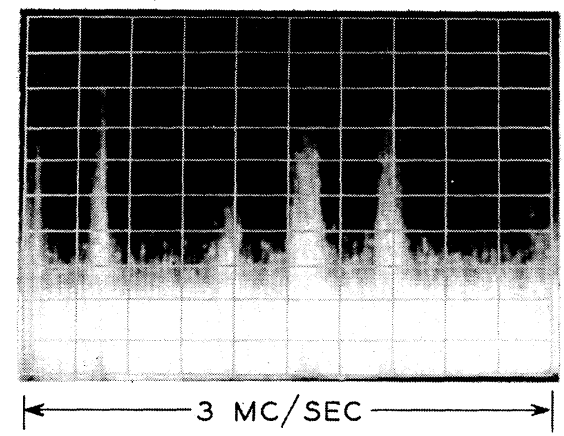

(a)

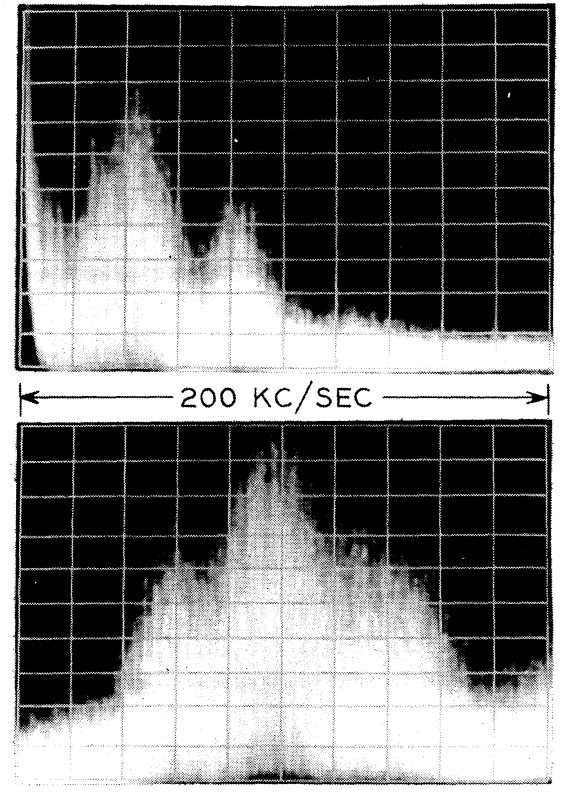

(b)

(c)

FIG. 4. Frequency spectra of the beat notes between various maser beam components. 
correlation was found between the visual structure of the beam observed through the infrared image converter, and the presence of the beats as the angular alignment of the plates was varied. Figure 4(a) shows several beat notes ranging from $50 \mathrm{kc} / \mathrm{sec}$ to $2 \mathrm{Mc} / \mathrm{sec}$. Figures $4(\mathrm{~b})$ and 4(c) show the structure of these beats on an expanded scale. It should be noted that in our present system the angular adjustment of the plates is also accompanied by a slight change in plate separation. The important conclusion to be drawn here regards the linewidth of the maser oscillation. As is evident from Figs. 4(b) and 4(c), the beat notes imply linewidths in the range from 10 to $80 \mathrm{kc} / \mathrm{sec}$.

The use of excitation transfer for production of inverted population was suggested ${ }^{1,2}$ by one of the authors. The He-Ne mixture described above is the first gaseous system which has led to maser oscillations at optical frequencies. Recently some evidence for the presence of inverted populations in a $\mathrm{Hg}-\mathrm{Zn}$ mixture has been reported ${ }^{11}$ which supports the wide applicability of this principle. ${ }^{1,2}$

In the course of this research, a number of co-workers have contributed in the design, construction, and measurement. We are indebted to F. Muller, P. S. Kubik, A. R. Strnad, D. MacNair,
E. Koch, G. J. Wolff, and G. E. Reitter. Dr. A. F. Turner of Bausch \& Lomb has been very helpful in supplying the special evaporated reflectance films. In particular we would like to acknowledge the expert and patient assistance given by $\mathrm{Mr}$. E. A. Ballik in the later stages of this experiment.

${ }^{1}$ A. Javan, Phys. Rev。 Letters $\underline{3}, 518$ (1959)。

${ }^{2}$ A. Javan, in Quantum Electronics, edited by C. H. Townes (Columbia University Press, New York, 1960).

${ }^{3}$ For simplicity, Paschen notation is used to describe the Ne energy levels.

${ }^{4}$ Not all of the $1 s$ levels are truly metastables: the $1 s_{2}$ and $1 s_{4}$ undergo transitions to the ground state; however, due to the trapping of the resonant ultraviolet photons these levels are effectively long-lived and quasi-metastable.

${ }^{5}$ M. Pahl and U. Weimer, Z. Naturforsch. 12a, 926 (1957).

${ }^{6}$ A. V. Phelps, Phys. Rev. 99, 4, 1307 (1955).

${ }^{7}$ W. R. Bennett, Jr。, A。 Javan, and E. A. Ballik, Bull. Am. Phys. Soc. 5, 496 (1960).

${ }^{8}$ R. Ladenberg, Revs. Modern Phys. 5, 243 (1933).

${ }^{9}$ A. L. Schawlow and C. H. Townes, Phys. Rev。112, 1940 (1958).

${ }^{10}$ A. G. Fox and P. Li, Proc. Inst. Radio Engrs. $\underline{48}$, 1904 (1960).

${ }^{11}$ V. K. Ablekov, M. S. Pesiu, and I. L. Fabelinski, Zhur. Eksp. i Teoret. Fiz。 39, 892 (1960).

\title{
MASS SPECTROGRAPHIC IDENTIFICATION OF THE ION OBSERVED IN HYDROGEN MOBILITY EXPERIMENTS*
}

\author{
W. S. Barnes, D. W. Martin, and E. W. McDaniel \\ Engineering Experiment Station and School of Physics, \\ Georgia Institute of Technology, Atlanta, Georgia
}

(Received January 9, 1961)

In a Letter appearing in a recent issue of this journal, Varney ${ }^{1}$ pointed out that previous studies of the mobility of ions in pure hydrogen have probably been misinterpreted. In the majority of experiments performed to date, only one ion has been observed, and it has commonly been thought to be $\mathrm{H}_{2}^{+}$. Indications of a second ion, generally assumed to be $\mathrm{H}^{+}$, have been seen in a few experiments but the actual existence of this ion remains in doubt. Upon consideration of a study by Stevenson and Schissler ${ }^{2}$ of the cross section for formation of $\mathrm{H}_{3}^{+}$by the reaction $\mathrm{H}_{2}^{+}+\mathrm{H}_{2} \rightarrow$ $\mathrm{H}_{3}^{+}+\mathrm{H}$, Varney has made the following assertions: (1) The $\mathrm{H}_{2}^{+}$ion is never observed in hydrogen mobility experiments; (2) the ion which in- variably appears in these experiments is $\mathrm{H}_{3}{ }^{+}$; (3) it is reasonable to assume that the second ion, if actually present, is $\mathrm{H}^{+}$. The purpose of this Letter is to report evidence which tends to corroborate Varney in each of these assertions.

We have recently constructed and put into operation apparatus designed to permit identification of the ions appearing in mobility experiments and to yield information concerning the various types of reactions which can occur at low energy between ions and molecules. ${ }^{3}$ Ions are produced by electron bombardment inside a long drift tube containing gas at a pressure up to about 700 microns. The ions diffuse down the drift tube under the influence of a weak electric 

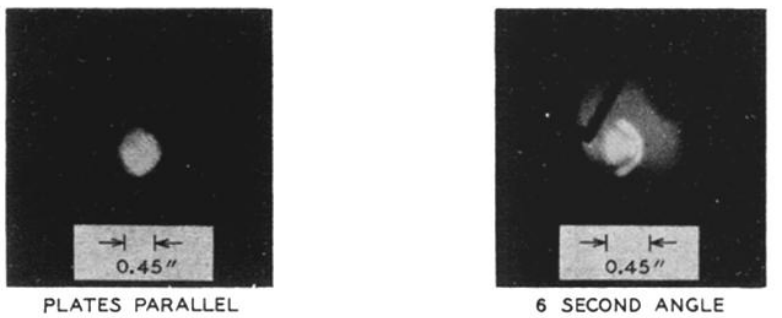

PATTERN IN MASER CAVITY
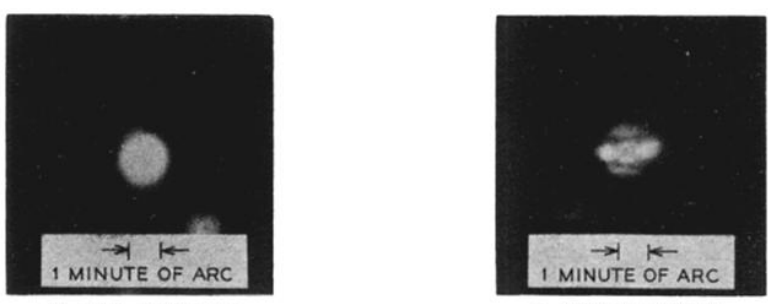

PLATES PARALLEL

6 SECOND ANGLE

ANGULAR DISTRIBUTION IN BEAM

FIG. 2. Far-field and near-field patterns from maser beam at two plate angles. 


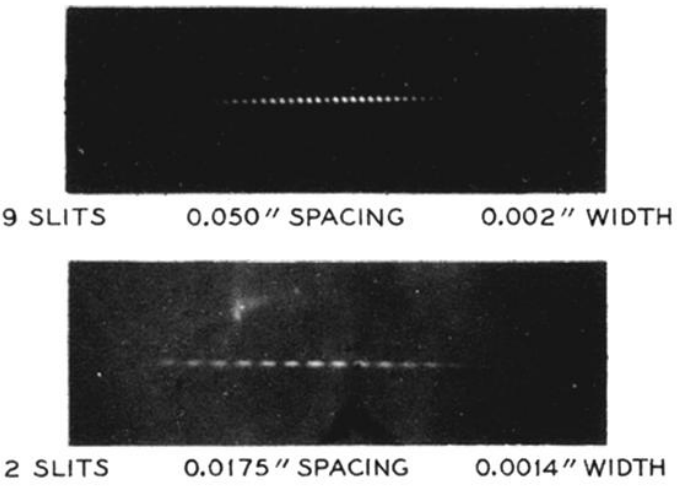

FIG. 3. Far-field diffraction patterns obtained by inserting multiple slits in the maser beam. 


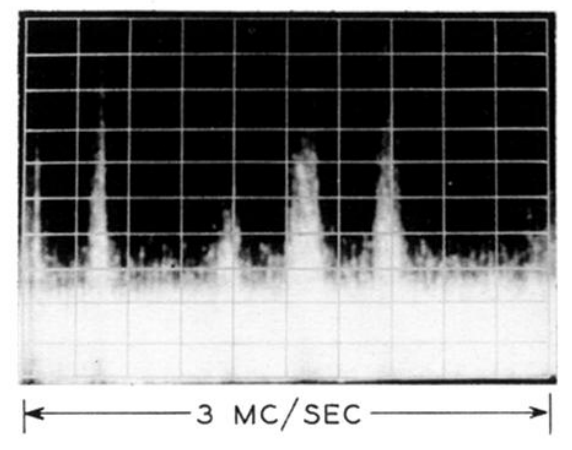

(a)

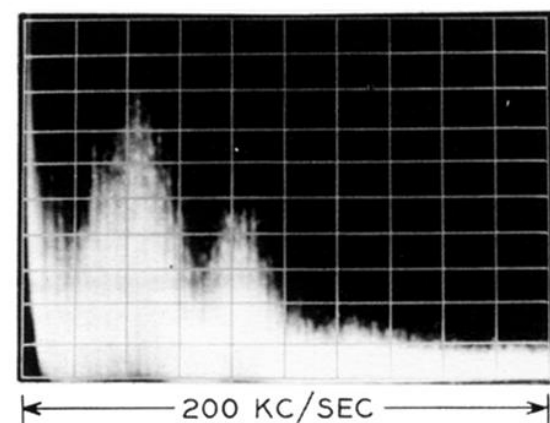

(b)

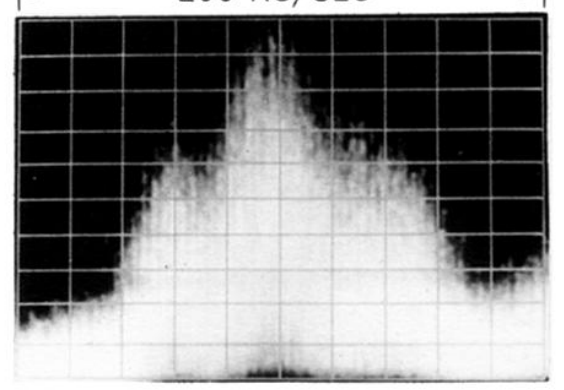

(c)

FIG. 4. Frequency spectra of the beat notes between various maser beam components. 